\title{
Radiation-Induced Peripheral Malignant Nerve Sheath Tumor Arising from Vestibular Schwannoma after Linac-Based Stereotactic Radiation Therapy: A Case Report and Review of Literatures
}

\author{
Putipun Puataweepong, ${ }^{1}$ Taweesak Janwityanujit, ${ }^{2}$ \\ Noppadol Larbcharoensub, ${ }^{3}$ and Mantana Dhanachai ${ }^{1}$ \\ ${ }^{1}$ Department of Radiology, Faculty of Medicine, Ramathibodi Hospital, Mahidol University, Bangkok 10400, Thailand \\ ${ }^{2}$ Department of Surgery, Faculty of Medicine, Ramathibodi Hospital, Mahidol University, Bangkok 10400, Thailand \\ ${ }^{3}$ Department of Pathology, Faculty of Medicine, Ramathibodi Hospital, Mahidol University, Bangkok 10400, Thailand
}

Correspondence should be addressed to Putipun Puataweepong, putipun.pua@mahidol.ac.th

Received 10 April 2012; Accepted 30 May 2012

Academic Editor: Aaron S. Dumont

Copyright (C) 2012 Putipun Puataweepong et al. This is an open access article distributed under the Creative Commons Attribution License, which permits unrestricted use, distribution, and reproduction in any medium, provided the original work is properly cited.

\begin{abstract}
In recent years the use of stereotactic radiation for vestibular schwannomas has increased worldwide. However, malignant transformation associated with radiation, although uncommon, has been reported in recent publications. We present a case of the 34 year-old female who had left vestibular schwannoma and who underwent surgery and postoperative stereotactic radiotherapy (SRT), hypofraction in 2005. At 6 years after SRT, the patient came with left facial palsy and severe headache. CT brain revealed progression in size with cystic and hemorrhagic changes of the preexisting tumor at left CPA with new obstructive hydrocephalus. Partial tumor removal was done, and the pathological report was malignant peripheral nerve sheath tumor (MPNST). Regarding the uncertainty of carcinogenesis risk, we should still practice radiation therapy with caution, especially in the young patient with tumor predisposition syndrome. Because of low incidence of MPNST after radiation, it should not be a major decision about giving radiotherapy. However, with the poor prognosis of MPNST, this possibility should be explained to the patient before radiation treatment option.
\end{abstract}

\section{Introduction}

In recent years, stereotactic radiosurgery (SRS) and fractionated stereotactic radiotherapy (SRT) have been considered the best management strategy for the majority of smallto-medium size vestibular schwannomas (VS) $[1,2]$. The widespread use of SRS/SRT in benign VS causes a great concern regarding radiation-induced malignancy, which is the rare but serious complication after radiation.

We reported a case with sporadic benign VS, which had transformed to malignant peripheral nerve sheath tumor (MPNST) after 6 years of SRT with a review of the literature.

\section{Case Report}

A 34-year-old female, without a familial history of neurofibromatosis, presented with left hearing disturbance. Magnetic resonance imaging (MRI) in 2004 showed VS at left cerebellopontine angle. Two times of left retrosigmoid approach with partial tumor removal were done during 2004. Pathological report was schwannoma WHO grade I. At 1 year after the second surgery, SRT, hypofraction was given due to regrowth of the tumor. Linear accelerator base system $(6$ MV dedicated Linac, Varian; with X-knife planning system version 3\&4, Radionics) was used for SRT treatment. Four 


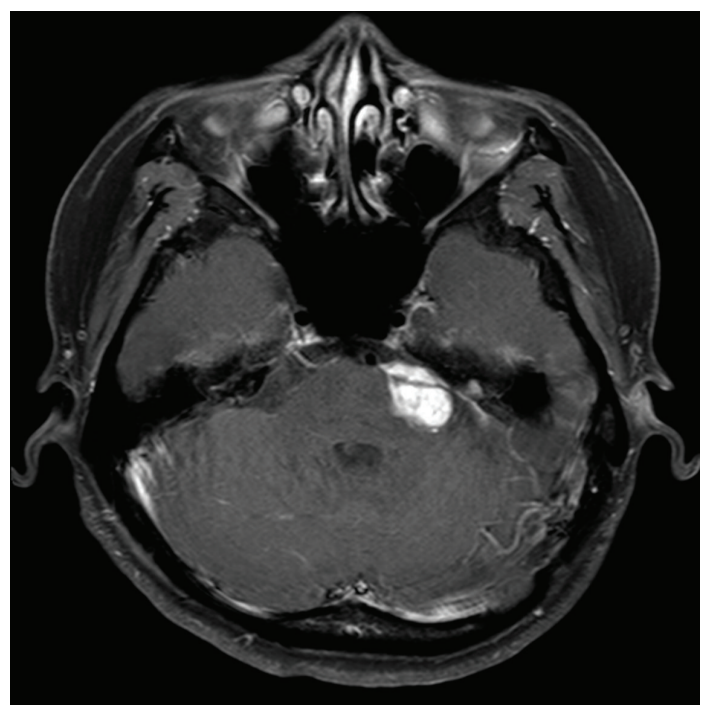

(a)

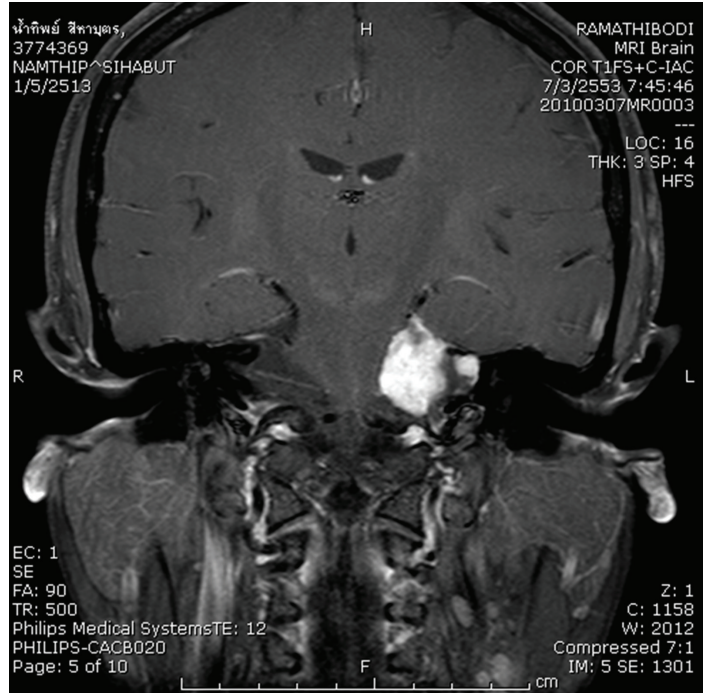

(b)

FIGURE 1: Axial and coronal T1W MRI with contrast showing a left vestibular schwannoma at the time before SRT (2005).
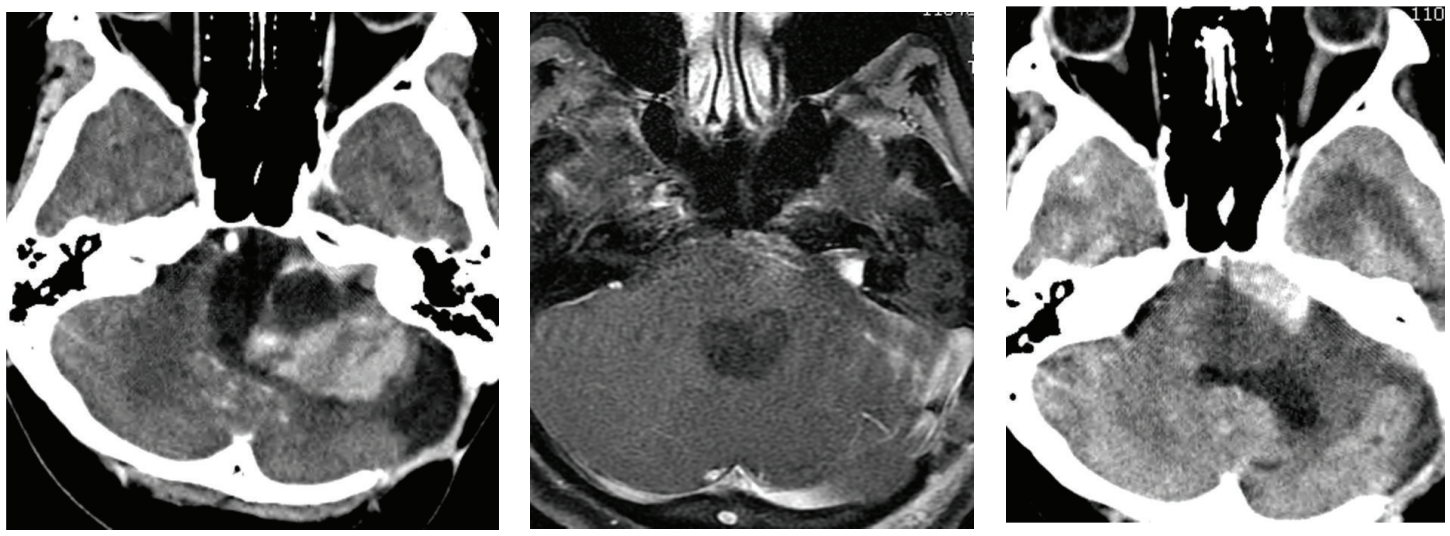

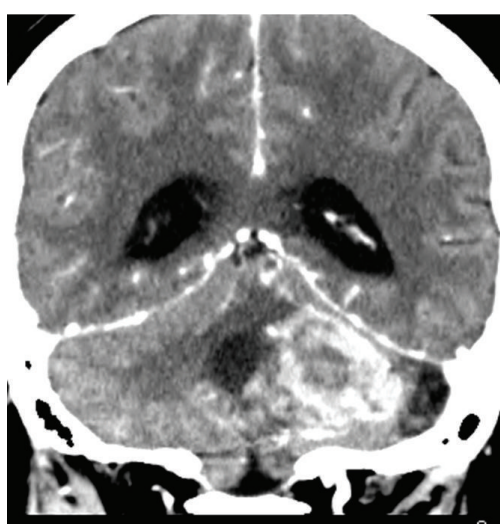

(a)

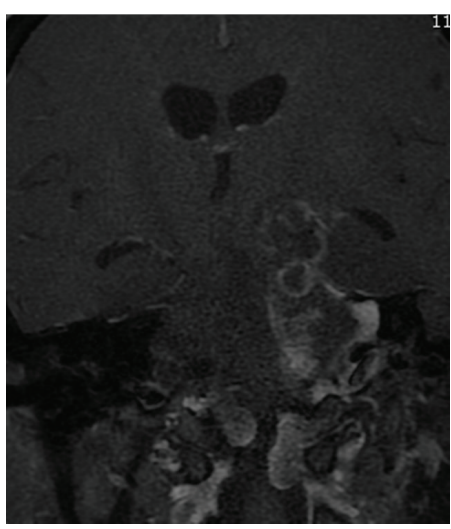

(b)

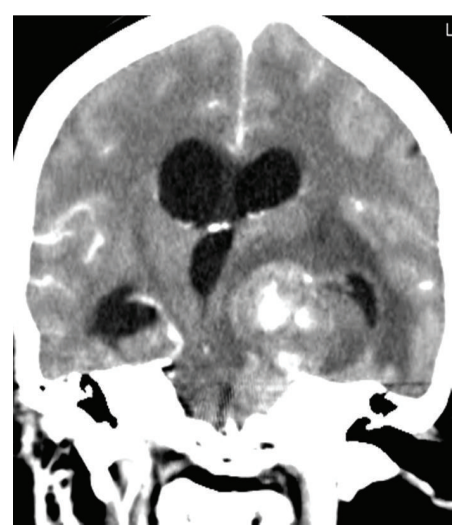

(c)

FIgURE 2: (a) Axial and Coronal CT with contrast showing radiological recurrence consistent with symptomatic recurrence 6 years after SRT (2011). (b) MRI was done at 3 weeks after recraniotomy with partial tumor removal. There was residual tumor with intense enhancement at the left internal auditory canal, measured about $0.9 \times 0.8 \times 1.5 \mathrm{~cm}^{3}$ in size, tumor volume measured about $0.535 \mathrm{cc}$. Newly seen was the cluster of hypointense T1/heterogeneous dark-hypointense T2 lesions with hyperintense T1 rim at the superior aspect of the surgical site, involving the left-sided prepontine cistern left-sided perimesencephalic and ambient cistern measured about $2.6 \times 1.6 \times 2.4 \mathrm{~cm}$ in AP, transverse and vertical greatest dimension. These findings were suggestive of the resolving hematomas. (c) The last CT scan of the patient. During 10 fractions of 3D-CRT, the patient developed deterioration of consciousness. CT scan showed rapid progression of residual tumor with internal bleeding. Obstructive hydrocephalus was more severe. 


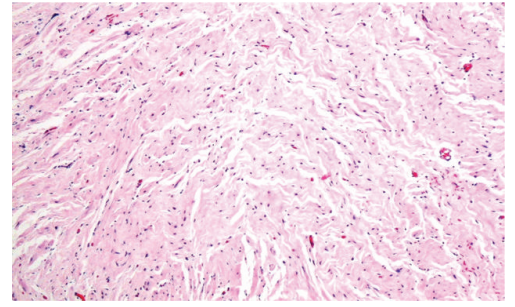

(a)

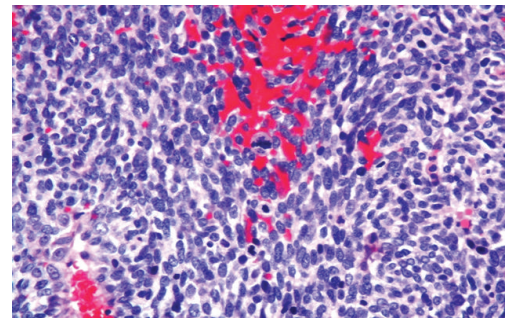

(d)

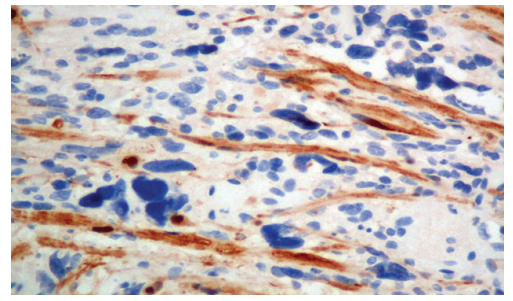

(g)

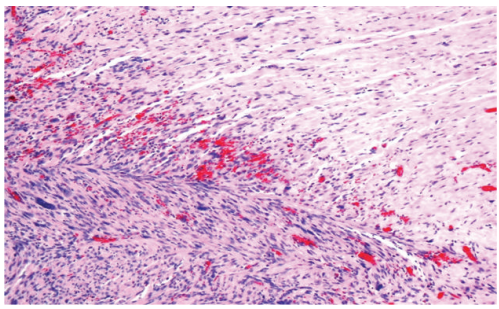

(b)

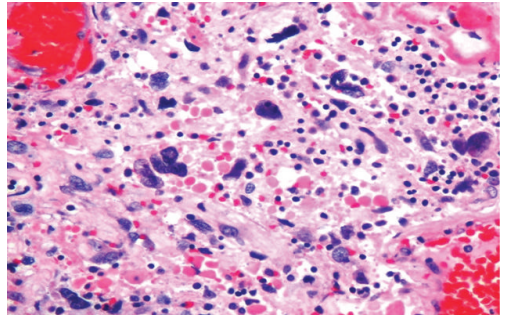

(e)

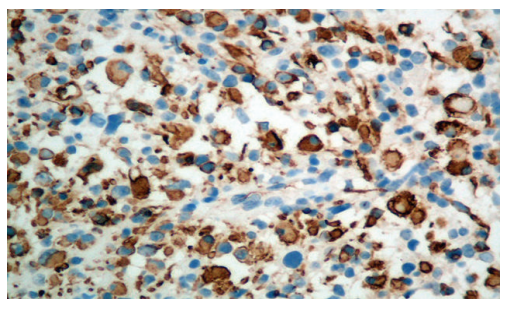

(h)

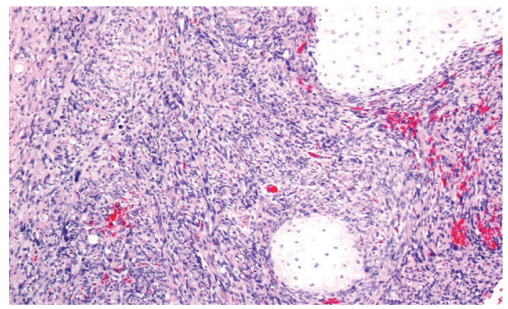

(c)

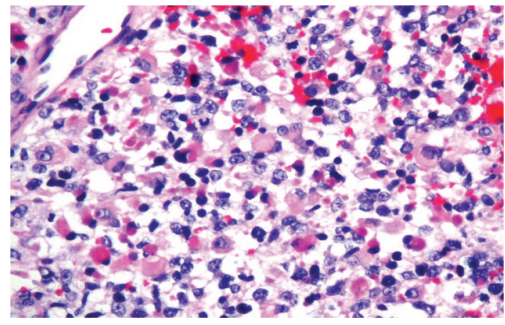

(f)

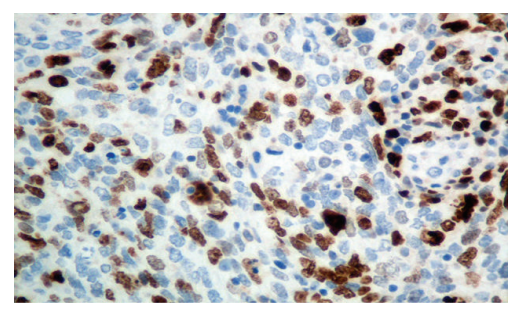

(i)

FIgURE 3: Histopathology of the first diagnosis of schwannoma $(1(\mathrm{a}), \times 40)$, histopathology of the following specimen showing malignant peripheral nerve sheath tumor (MPNST) arising in schwannoma (1(b), $\times 40)$, MPNST with chondrosarcomatous component (1(c), $\times 40)$, MPNST with area composed of round cell tumor and brisk mitotic activity $(1(\mathrm{~d}), \times 100)$, MPNST with pleomorphic nuclei $(1(\mathrm{e}), \times 400)$, MPNST with rhabdomyoblastic differentiation (1(f), $\times 400)$, MPNST showing immunonegativity for S-100 (1(g), X400), MPNST showing sarcomeric actin immunoreactivity $(1(\mathrm{~h}), \times 400)$, and MPNST with high Ki-67 immunohistochemistry $(1(\mathrm{i}), \times 400)$.

isocenters with total average dose of 30 Gray in 6 fractions prescribed at $80 \%$ isodose line were given to the patient during 2 weeks period. The tumor volume was $4.8 \mathrm{cc}$. The tumor had been well controlled for 6 years after SRT. Figure 1 revealed the MRI Axial and Coronal showing a left VS at the time before SRT.

The patient presented with left facial palsy, dysphagia, and right hemiparesis 6 years after SRT. Computer tomography revealed marked progression in size with cystic and hemorrhagic changes of the preexisting tumor at left CPA with new obstructive hydrocephalus (Figure 2(a)). Craniotomy with tumor removal was done immediately. MRI was performed at 3 weeks after operation, and the study shows small residual tumor at the left internal auditory canal with the cluster lesions at the superior aspect of the surgical site that is compatible with the resolving hematomas (Figure 2(b)). The patient had improvement of her neurological symptom after surgery. The pathological report was malignant peripheral nerve sheath tumor arising on schwannoma, with heterogenous mesenchymal rhabdomyoblastic and chondroblastic differentiation, WHO grade IV.

Because of malignant histology and small residual tumor, the patient underwent postoperative radiation therapy with 3-dimensional conformal radiation therapy (3D-CRT). A total dose of 54 Gray in 27 fractions was planned for the patient. After 20 Gray in 10 fractions of 3D-CRT, the patient developed deterioration of consciousness. CT emergency was done and showed progression of residual tumor with internal bleeding (Figure 2(c)). Obstructive hydrocephalus was more severe. Ventriculoperitoneal shunt was performed immediately. After VP shunt, the patient still had no cooperation and showed no response to deep pain. Because the prognosis of the patient was poor, we discussed with her husband about the end of life care. Finally, the patient was discharged home for best supportive care 1 month later.

2.1. Histology. The two pathological specimens were examined in two occasions as shown in Figure 3. The first specimen reveals low cellularity spindle cell tumor with minimal nuclear atypia and fibrillary cytoplasm that are compatible with schwannoma (Figure 3(a)).

The second specimen was performed after irradiation and composed of markedly hypercellularity and hyperchromatic spindle cells growing in fasciculated pattern. The cytoplasm is typically light staining and indistinct (Figure 3(b)). A few areas of markedly pleomorphic nuclei, brisk mitotic 
TABLE 1: The cases of malignant transformation of VS after stereotactic radiation.

\begin{tabular}{|c|c|c|c|c|c|}
\hline Author & Age/sex & NF2 & RT tech & Final pathology & Years to 2nd tumor \\
\hline Thomsen et al., 2000 [3] & $19 / \mathrm{F}$ & $\mathrm{Y}$ & SRS & Sarcoma & 6 \\
\hline Baser et al., $2000[4]$ & N/A & $\mathrm{Y}$ & SRS & $\begin{array}{c}3 \text { MPNSTs } \\
1 \text { malignant meningioma, } \\
1 \text { malignant ependymoma } \\
\text { (de novo) }\end{array}$ & N/A \\
\hline Ho and Kveton, 2002 [5] & $14 / \mathrm{F}$ & $\mathrm{Y}$ & SRS & Rapid growth (no patho) & 7 months \\
\hline McEvoy and Kitchen, 2003 [6] & $22 / \mathrm{M}$ & $\mathrm{Y}$ & SRS & Rapid growth (no patho) & 2 \\
\hline Bari et al., 2002 [7] & $28 / \mathrm{F}$ & $\mathrm{Y}$ & SRS & MPNST & 4 \\
\hline Rowe et al., 2008 [8] & $\mathrm{F}$ & Y & SRS & Malignant glioma (de novo) & 3 \\
\hline Carlson et al., 2010 [9] & $25 / \mathrm{F}$ & $\mathrm{Y}$ & SRT & Rhabdosarcoma (de novo) & 10 \\
\hline Husseini et al., $2011[10]$ & $20 / \mathrm{M}$ & $\mathrm{Y}$ & SRS & MPNST & 5 \\
\hline Comey et al., 1998 [11] & $50 / \mathrm{M}$ & $\mathrm{N}$ & SRS & Triton & 5 \\
\hline Shamisa et al., 2001 [12] & $57 / \mathrm{F}$ & $\mathrm{N}$ & SRS & GBM (de novo) & 7.5 \\
\hline Hanabusa et al., $2001[13]$ & $51 / \mathrm{F}$ & $\mathrm{N}$ & SRS & Sarcoma & 6 months \\
\hline Shin et al., 2002 [14] & $26 / \mathrm{F}$ & $\mathrm{N}$ & SRS & MPNST & 6 \\
\hline Wilkinson et al., 2004 [15] & $53 / \mathrm{M}$ & $\mathrm{N}$ & SRS & MPNST & 4 \\
\hline Muracciole et al., 2004 [16] & $61 / \mathrm{F}$ & $\mathrm{N}$ & SRS & Triton & 6 \\
\hline Maire et al., 2006 [17] & N/A & $\mathrm{N}$ & SRT & MPNST & 19 \\
\hline Balasubramaniam et al., 2007 [18] & $64 / \mathrm{F}$ & $\mathrm{N}$ & SRT & GBM (de novo) & 5 \\
\hline Yang et al., 2010 [19] & $74 / \mathrm{M}$ & $\mathrm{N}$ & SRS & Sarcoma & 6 \\
\hline Demetriades et al., 2010 [20] & $37 / \mathrm{M}$ & $\mathrm{N}$ & SRS & MPNST & 10 \\
\hline Akamatsu et al., 2010 [21] & $67 / \mathrm{F}$ & $\mathrm{N}$ & SRS & MPNST & 7.5 \\
\hline Our case 2011 & $34 / \mathrm{F}$ & $\mathrm{N}$ & SRT & MPNST & 6 \\
\hline
\end{tabular}

activity, and round cells epithelioid appearance are also seen (Figures 3(d) and 3(e)).

The capacity of MPNST to undergo focal mesenchymal differentiation is well known. Rhabdomyosarcoma is most frequent. The other sarcomatous component may be present such as chondrosarcoma and osteosarcoma. In this case, pluridirectional differentiations are observed as chondrosarcomatous component (Figure 3(c)) and rhabdomyoblastic component (Figure 3(f)).

Immunohistochemically, S-100 is negative (Figure 3(g)) and confirms rhabdoid/skeletal muscle differentiation with positive staining with sarcomeric actin (Figure $3(\mathrm{~h})$ ). The high proliferative index ( $\mathrm{Ki} 67$ ) is also seen supporting malignant behavior (Figure 3(i)).

\section{Discussion}

Stereotactic radiosurgery (SRS) or fractionated stereotactic radiotherapy (SRT) is techniques to administer precisely directed, high-dose irradiation that tightly conforms to an intracranial target to create a desired radiobiologic response while minimizing radiation dose to the surrounding normal tissues. In recent years, SRS/SRT has become a commonly used modality for brain tumor especially in VS treatment. Although radiation-induced malignant brain tumors possibly from this technique have been reported, there were only few studies with complete review. The purpose of our study is, therefore, to present another case reports in the radiationinduced MPNST with an extensive review of literatures.

In 1948, Cahan et al. [22] established the criteria of a radiation-induced tumor which included (1) a second tumor occurs within the radiation field, and it was not present at the time of radiation, (2) a latency period is required between radiotherapy and tumor development (several years), (3) a histological difference must exist between the primary and the new tumor, and (4) the patient should not have any genetic predisposition for cancer development. In this report, it seems that our case might not fulfill all of the Cahan's criteria by some reasons. Firstly, a primary and the new tumor do not have a completely histological difference. Secondly, there was only $1 \mathrm{~mL}$ of tumor tissue from ultrasonic aspirator sent for pathological section which may not sufficiently represent the rest of tumor. Thirdly, we found that there were some areas of increased vascularity and adhesion to the upper brainstem causing difficulty in tumor removal during the first two operations which may be signs of a higher than grade 1 tumor. However, we believe that it is still reasonable to consider or suspect that radiation effects from SRS/SRT have had a major role in the pathogenesis of a malignant conversion from the primary benign tumor into MPNST in this patient.

The incidence of MPNST is very rare, approximately $0.001 \%$ [23]. Most cases developed sporadically (50\%), $30 \%$ arising from malignant transformation of benign schwannoma, and radiation is a well-established carcinogen 
and associated with malignant transformation of vestibular schwannoma (VS). Comey et al. [11] described that, after radiation, most of the irradiated cells usually undergo cytoplasmic vacuolization and subsequent cell death, Rarely, some of the surviving cells might acquire genetic mutations, which are responsible for the malignant transformation of VS. From literature reviews, we found 24 [3-21] (including our report) cases of malignant brain tumor following stereotactic radiotherapy of VS (Table 1). There was 16 cases of malignant transformation of VS. De novo secondary tumor (the new tumor that appeared in the radiation field) was found in 6 cases, and 2 patients had rapid growth of tumor several years after radiation. Most of them received SRS and had histological proof of a malignant tumor after radiation (20 cases). Twelve cases were neurofibromatosis 2 (NF-2). The median time to develop secondary tumor was 6 years.

NF-2 is a rare $(1: 40,000)$ autosomal dominant disease that is caused by mutation of the NF2 tumor suppressor gene. From our reviews, we found a high incidence of malignant transformation in irradiated NF-2 patients (52\%). Baser et al. [4] surveyed $1348 \mathrm{NF}-2$ patients and reported that the incidence of malignant transformation was $10 \%$ in the irradiated patient compared to $0.7 \%$ in the nonirradiated. They concluded that NF-2 patients had a 14 -fold increased risk of developing malignant brain tumor after radiation. Regarding the higher incidence of radiation-induced malignant transformation, observation and surgery are the preferred treatment option for NF-2 patients, particularly for those who are young.

After radiation exposure, there are two possible outcomes. One is the deterministic effect, which has a threshold of dose and a severity of the effects which are dose related. Another effect is called stochastic, which is carcinogenesis, hereditary effects fall in this category. If somatic cells are exposed to radiation, the probability of cancer increases with dose, probably with no threshold. But the severity of the cancer is not dose related. A cancer induced by 1 Gray is no worse than one induced by 0.1 Gray. But of course the probability of its induction is increased. An advanced conformal radiation technique, such as 3D-CRT, IMRT or SRS/SRT, can deliver high radiation dose to tumor with lower dose to normal tissue. This might reduce only the deterministic effect, but the effect to the stochastic is still unknown. Regarding the uncertainty of carcinogenesis risk, we should still practice radiation therapy with caution, especially in young patients with tumor predisposition syndrome. Because of low incidence of MPNST after radiation, it should not be a major decision about giving radiotherapy. However, with the poor prognosis of MPNST, this possibility should be explained to the patient before radiation treatment option.

\section{References}

[1] B. E. Pollock, "Vestibular schwannoma management: an evidence-based comparison of stereotactic radiosurgery and microsurgical resection," Progress in neurological surgery, vol. 21, pp. 222-227, 2008.
[2] C. E. Park, B. J. Park, Y. J. Lim, and S. G. Yeo, "Functional outcomes in retrosigmoid approach microsurgery and gamma knife stereotactic radiosurgery in vestibular schwannoma," European Archives of Oto-Rhino-Laryngology, vol. 268, no. 7, pp. 955-959, 2011.

[3] J. Thomsen, F. Mirz, R. Wetke, J. Astrup, M. Bojsen-Møller, and E. Nielsen, "Intracranial sarcoma in a patient with neurofibromatosis type 2 treated with gamma knife radiosurgery for vestibular schwannoma," American Journal of Otology, vol. 21, no. 3, pp. 364-370, 2000.

[4] M. E. Baser, D. G. R. Evans, R. K. Jackler, E. Sujansky, and A. Rubenstein, "Neurofibromatosis 2, radiosurgery and malignant nervous system tumours," British Journal of Cancer, vol. 82, no. 4, p. 998, 2000.

[5] S. Y. Ho and J. F. Kveton, "Rapid growth of acoustic neuromas after stereotactic radiotherapy in type 2 neurofibromatosis," Ear, Nose and Throat Journal, vol. 81, no. 12, pp. 831-833, 2002.

[6] A. W. McEvoy and N. D. Kitchen, "Rapid enlargement of a vestibular schwannoma following gamma knife treatment," Minimally Invasive Neurosurgery, vol. 46, no. 4, pp. 254-256, 2003.

[7] M. E. Bari, D. M. C. Forster, A. A. Kemeny, L. Walton, D. Hardy, and J. R. Anderson, "Malignancy in a vestibular schwannoma. Report of a case with central neurofibromatosis, treated by both stereotactic radiosurgery and surgical excision, with a review of the literature," British Journal of Neurosurgery, vol. 16, no. 3, pp. 284-289, 2002.

[8] J. Rowe, M. Radatz, and A. Kemeny, "Radiosurgery for type II neurofibromatosis," Progress in neurological surgery, vol. 21, pp. 176-182, 2008.

[9] M. L. Carlson, D. Babovic-Vuksanovic, L. N. E. Messiaen, B. W. Scheithauer, B. A. Neff, and M. J. Link, "Radiationinduced rhabdomyosarcoma of the brainstem in a patient with neurofibromatosis Type 2: case report," Journal of Neurosurgery, vol. 112, no. 1, pp. 81-87, 2010.

[10] S. T. Husseini, E. Piccirillo, A. Taibah, C. T. Paties, R. Rizzoli, and M. Sanna, "Malignancy in vestibular schwannoma after stereotactic radiotherapy: a case report and review of the literature," Laryngoscope, vol. 121, no. 5, pp. 923-928, 2011.

[11] C. H. Comey, M. R. Mclaughlin, H. D. Jho, A. J. Martinez, and L. D. Lunsford, "Death from a malignant cerebellopontine angle triton tumor despite stereotactic radiosurgery. Case report," Journal of Neurosurgery, vol. 89, no. 4, pp. 653-658, 1998.

[12] A. Shamisa, M. Bance, S. Nag et al., "Glioblastoma multiforme occurring in a patient treated with gamma knife surgery: case report and review of the literature," Journal of Neurosurgery, vol. 94, no. 5, pp. 816-821, 2001.

[13] K. Hanabusa, A. Morikawa, T. Murata, and W. Taki, "Acoustic neuroma with malignant transformation. Case report," Journal of Neurosurgery, vol. 95, no. 3, pp. 518-521, 2001.

[14] M. Shin, K. Ueki, H. Kurita, and T. Kirino, "Malignant transformation of a vestibular schwannoma after gamma knife radiosurgery," Lancet, vol. 360, no. 9329, pp. 309-310, 2002.

[15] J. S. Wilkinson, H. Reid, and G. R. Armstrong, "Malignant transformation of a recurrent vestibular schwannoma [4]," Journal of Clinical Pathology, vol. 57, no. 1, pp. 109-110, 2004.

[16] X. Muracciole, D. Cowen, and J. Régis, "Radiosurgery and brain radio-induced carcinogenesis: update," Neurochirurgie, vol. 50, no. 2-3, pp. 414-420, 2004.

[17] J. P. Maire, A. Huchet, Y. Milbeo et al., “Twenty years' experience in the treatment of acoustic neuromas with fractionated radiotherapy: a review of 45 cases," International Journal of 
Radiation Oncology Biology Physics, vol. 66, no. 1, pp. 170-178, 2006.

[18] A. Balasubramaniam, P. Shannon, M. Hodaie, N. Laperriere, H. Michaels, and A. Guha, "Glioblastoma multiforme after stereotactic radiotherapy for acoustic neuroma: case report and review of the literature," Neuro-Oncology, vol. 9, no. 4, pp. 447-453, 2007.

[19] T. Yang, J. Rockhill, D. E. Born, and L. N. Sekhar, "A case of high-grade undifferentiated sarcoma after surgical resection and stereotactic radiosurgery of a vestibular schwannoma," Skull Base, vol. 20, no. 3, pp. 179-183, 2010.

[20] A. K. Demetriades, N. Saunders, P. Rose et al., "Malignant transformation of acoustic neuroma/vestibular schwannoma 10 years after gamma knife stereotactic radiosurgery," Skull Base, vol. 20, no. 5, pp. 381-387, 2010.

[21] Y. Akamatsu, K. Murakami, M. Watanabe, H. Jokura, and T. Tominaga, "Malignant peripheral nerve sheath tumor arising from benign vestibular schwannoma treated by gamma knife radiosurgery after two previous surgeries: a case report with surgical and pathological observations," World Neurosurgery, vol. 73, no. 6, pp. 751-754, 2010.

[22] W. G. Cahan, H. Q. Woodard, N. L. Higinbotham, F. W. Stewart, and B. L. Coley, "Sarcoma arising in irradiated bone: report of eleven cases. 1948," Cancer, vol. 82, no. 1, pp. 8-34, 1998.

[23] A. Ziadi and I. Saliba, "Malignant peripheral nerve sheath tumor of intracranial nerve: a case series review," Auris Nasus Larynx, vol. 37, no. 5, pp. 539-545, 2010. 


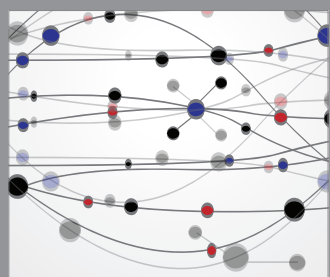

The Scientific World Journal
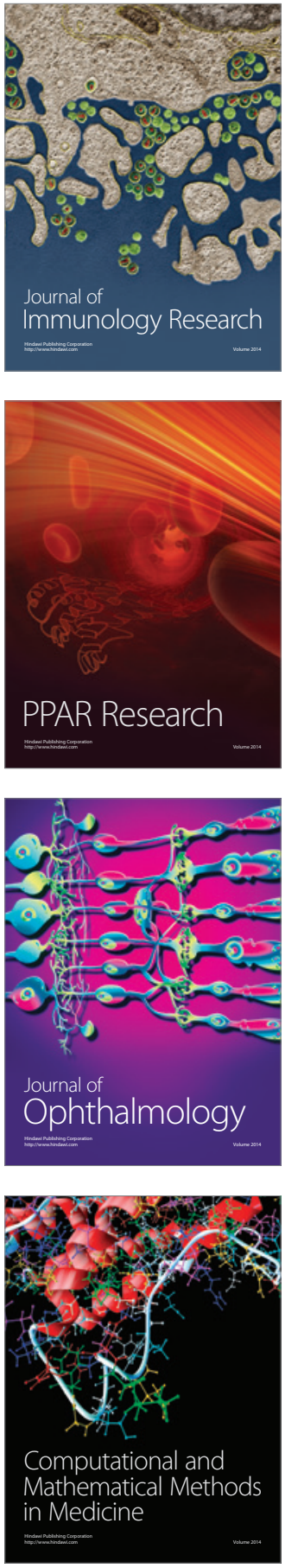

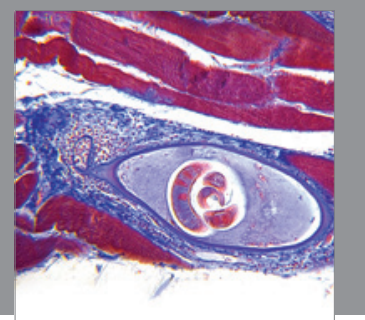

Gastroenterology

Research and Practice
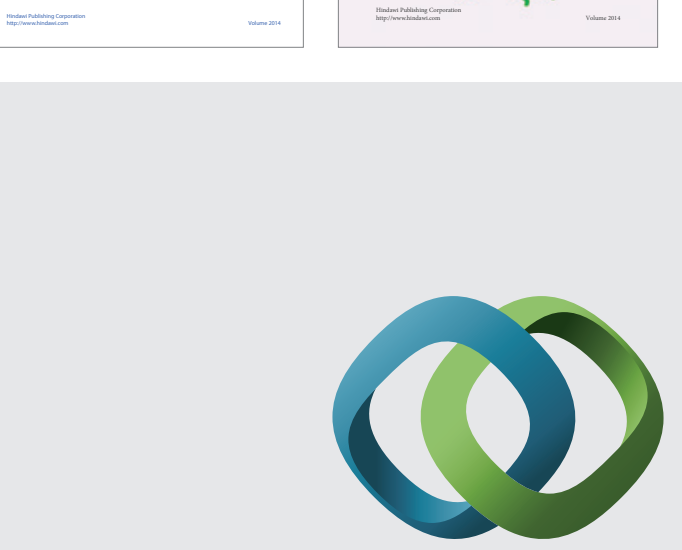

\section{Hindawi}

Submit your manuscripts at

http://www.hindawi.com
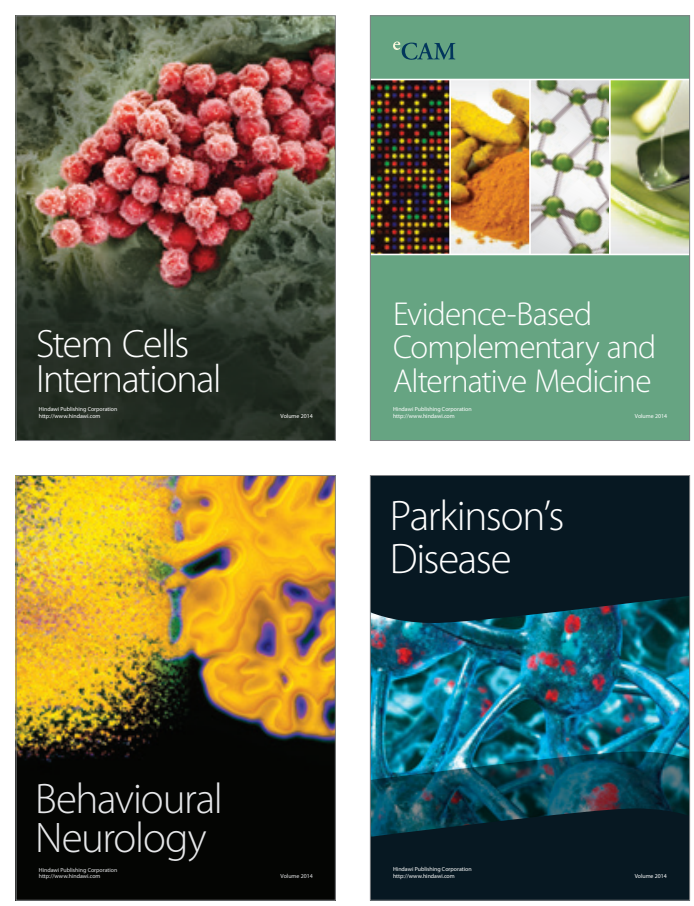

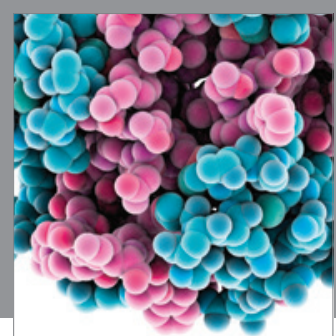

Journal of
Diabetes Research

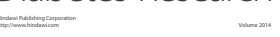

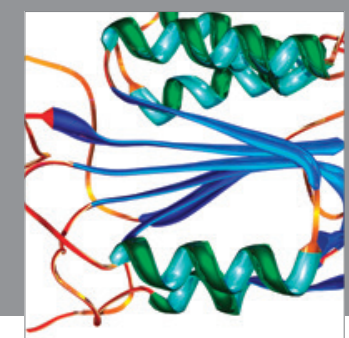

Disease Markers
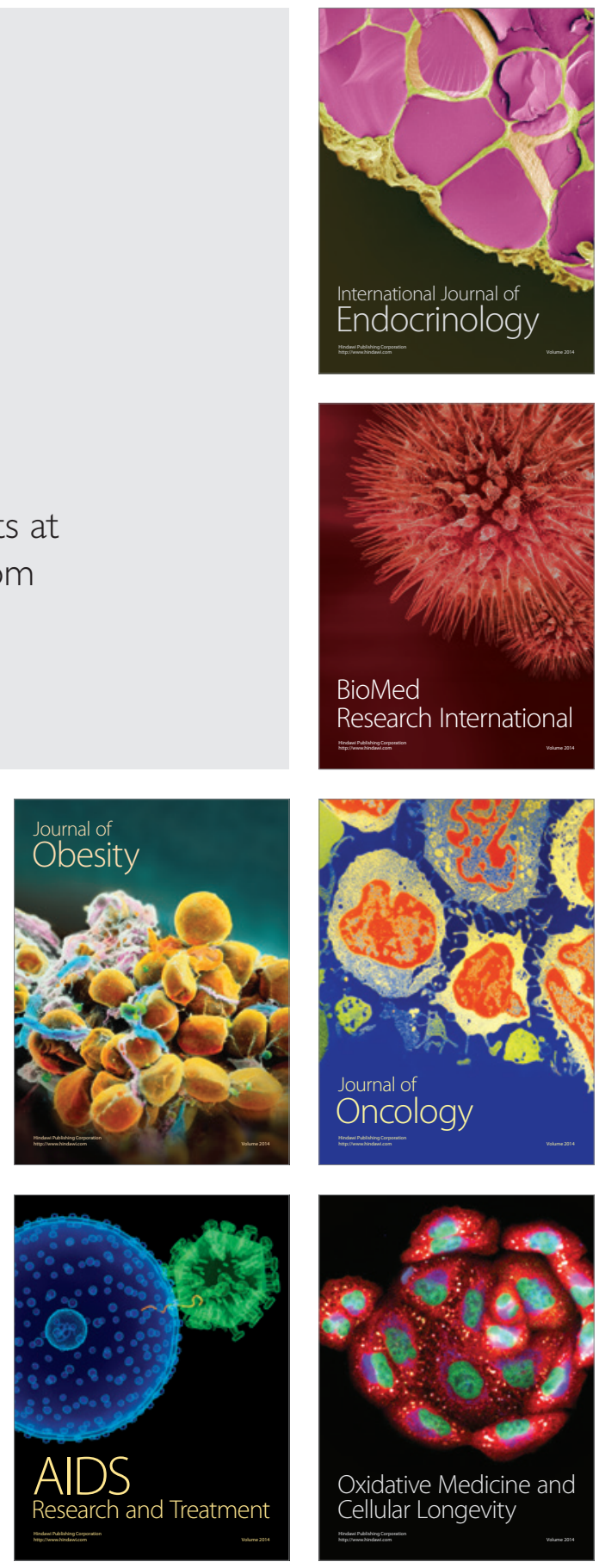